\title{
Comparison of Calcium Sulfate and Bovine Collagen Barriers for Alveolar Ridge Augmentation
}

\author{
${ }^{1}$ Matthew L Heaton, ${ }^{2}$ David G Kerns, ${ }^{3}$ William W Hallmon, ${ }^{4}$ Harvey P Kessler, ${ }^{5}$ Robert Spears \\ ${ }^{6}$ Eric S Solomon, ${ }^{7}$ Ibtisam Al-Hashimi
}

\begin{abstract}
Calcium sulfate is a biologically compatible osteoconductive graft material that binds underlying bone graft and provide space maintenance. The purpose of this study was to evaluate calcium sulfate as a barrier compared to a collagen membrane for augmentation of a standardized surgically created ridge defect. For this purpose, bilateral extraction of mandibular premolars was performed on six foxhounds (Canis familiaris). Eight weeks later, a standardized osseous ridge defects (24 total) were created using a $6 \mathrm{~mm}$ trephine. The study was approved by the Institutional Animal Care and Use Committee (IACUC) at Baylor College of Dentistry. The osseous defects were allocated into three groups (8 defects each): group 1 received autogenous bone graft covered with collagen membrane (CM); group 2 received autogenous bone graft covered with calcium sulfate barrier (CS), and group 3 was used as control and did not receive bone augmentation and was used as control. The animals were sacrificed after 12 weeks following bone augmentation and sites were evaluated histologically for total ridge width, percentage of bone gain and cortical bone thickness.
\end{abstract}

Results: All sites exhibited bony fill within the defect. Analysis of variance did not reveal statistically significant difference in the mean total bone gain among CM, CS, and control groups $(12.2,11.6$, and 11.9$) \mathrm{mm}^{2}$, respectively, $\mathrm{p}=0.875$.

Conclusion: Calcium sulfate does not appear to improve bone regeneration in an osseous defect.

Keywords: Guided bone regeneration, Ridge augmentation, Calcium sulfate, Collagen membrane.

How to cite this article: Heaton ML, Kerns DG, Hallmon WW, Kessler HP, Spears R, Solomon ES, Al-Hashimi I. Comparison of

\footnotetext{
${ }^{1}$ Private Practitioner, ${ }^{2}$ Professor and Director, ${ }^{3}$ Regents Professor (Retired), ${ }^{4-7}$ Professor

${ }^{1}$ Private Practice, Department of Graduate Periodontics Knoxville, Tennessee, USA

2,3,7Department of Periodontics, Texas A\&M University, Baylor College of Dentistry, Dallas, Texas, USA

${ }^{4}$ Department of Oral Pathology, Texas A\&M University, Baylor College of Dentistry, Dallas, Texas, USA

${ }^{5}$ Department of Biomedical Sciences, Texas A\&M University Baylor College of Dentistry, Dallas, Texas, USA

${ }^{6}$ Department of Administration, Texas A\&M University, Baylor College of Dentistry, Dallas, Texas, USA
}

Corresponding Author: David G Kerns, Professor and Director Department of Periodontics, Texas A\&M University, Baylor College of Dentistry, Dallas, Texas, USA, Phone: 2148288140, e-mail: dkerns@bcd.tamhsc.edu
Calcium Sulfate and Bovine Collagen Barriers for Alveolar Ridge Augmentation. J Contemp Dent 2014;4(3):129-138.

Source of support: Nil

Conflict of interest: The authors have no financial interest, either directly or indirectly, in the products $\left(\mathrm{Ossix}^{\mathrm{TM}}\right.$, Calcigen $^{\mathrm{TM}}$ Oral) evaluated in this study.

\section{INTRODUCTION}

Tooth extraction can result in a loss of up to $50 \%$ of socket alveolar bone in both a horizontal and vertical dimension. ${ }^{1}$ Fugazzotto ${ }^{2}$ reported that progressive atrophy of the residual alveolar ridge occurs up to 12 months following extraction.

Osseous grafting of a deficient alveolar ridge involves placement of a bone graft within the alveolar defect and a barrier to cover and protect the grafted bone. ${ }^{3,4}$ Buser, was one of the first to report successful ridge augmentation with guided bone regeneration (GBR) in humans using an expanded polytetrafluoroethylene (ePTFE) membrane and tenting pins. ${ }^{5}$ Becker et al reported bone regeneration of a alveolar ridge deficiencies using ePTFE and resorbable pins. ${ }^{6} \mathrm{GBR}$ as a ridge augmentation procedure can be accomplished using particulate bone, however, corticocancellous block grafts are useful for augmentation of severely resorbed alveolar ridge defects. ${ }^{7,8}$ Other alternatives to augmentation include distraction osteogenesis and use of growth factors, such as platelet-rich plasma or recombinant human bone morphogenic protein-2.9-12

The use of a resorbable membrane is common in ridge augmentation procedures, primarily due to the advantages of one surgical procedure vs multiple surgeries involved with placement of nonresorbable membranes. ${ }^{13-15}$ One of the major problems associated with the use of the ePTFE membrane is wound healing following exposure of the membrane, which often leads to localized tissue necrosis or infection requiring removal of the membrane. ${ }^{16,17}$ It has been reported that early exposure of a membrane may result in a less successful outcome in GBR procedures. ${ }^{18}$ Resorbable membranes tolerate poor vascularity and exposure better than nonresorbable membranes, with secondary epithelialization occurring over resorbable membrane exposures in most cases. ${ }^{19,20}$ Collagen-based membranes are usually degraded and incorporated into the surrounding periodontium within 
3 to 6 months following placement, which allows adequate time for osseous grafting materials to mature. ${ }^{19-21}$ Modification of collagen fibers known as cross-linking can prolong the rate of degradation. ${ }^{21}$ Collagen-based membranes have been found to be comparable to e-PTFE membranes in the management of extraction socket and furcation defects. ${ }^{22,23}$ Use of collagen membranes in ridge augmentation procedures is primarily limited to case reports, however current reports show promising findings in terms of quantity and quality of bone regenerate enabling dental implant placement. ${ }^{19,20,24}$

An alternative to ePTFE or collagen-based membranes for use in GBR procedures is calcium sulfate (CS). ${ }^{25,26}$ Medical grade calcium sulfate is a chemical compound that has been used for hard-tissue augmentation in the medical field for decades. ${ }^{27,28}$ Calcium sulfate is a biologically compatible graft material that is osteo-conductive, acting as a natural scaffold to preserve bone volume and allow proliferation of osteoprogenitor cells. An in vitro study by Payne et al compared fibroblast migration over different barrier materials found a significantly greater migration rate over CS compared to PTFE and polylactic acid. ${ }^{29}$ Sottosanti advocated the use of CS in conjunction with a bone grafting material as a composite graft, with the CS acting as a stabilizer and a binder of the osseous particles. ${ }^{25}$ Histological analysis of biopsies taken from extraction sites grafted with CS showed almost complete resorption of the material within 3 months of placement, and bone fill was greater compared with non-grafted control sites. ${ }^{30}$

Additional benefits beyond osteoconductivity and space maintenance have been found related to potential angiogenesis as a result of CS implantation. In a study of microvessel density within grafted osseous defects in rabbit tibiae, Strocchi et al reported that defects filled with CS and covered with ePTFE membrane had higher vascular density than those filled with CS or autologous bone only. ${ }^{31}$ These findings add additional support to the use of CS in dental regenerative procedures.

The use of CS in regeneration procedures is a practical adjunct to osseous grafting, however, the application of the material in GBR procedures is not widely accepted. Clinical studies that have used CS in ridge preservation procedures, endodontic surgery, dental implants and sinus augmentation add evidence supporting the use of CS as a graft material in GBR procedures. ${ }^{25,32-37}$

The purpose of this study was to compare bone regeneration using a bovine collagen membrane (CM) and CS as a barrier for ridge augmentation.

\section{MATERIALS AND METHODS}

The experimental model used in this study involved the creation of standardized alveolar ridge defects followed by GBR using osseous grafting and barrier devices in a canine model. Study protocol was approved by the Institutional Animal Care and Use Committee at Baylor College of Dentistry-a member of the Texas A\&M University Health Science Center.

\section{STUDY ANIMALS}

Six female American foxhounds (Canis familiaris) approximately 2 years old and weighing 25 to $30 \mathrm{~kg}$ were used in this study. All animals were subjected to a physical and dental examination and were quarantined for 10 days upon arrival. The protocols for anesthesia, postoperative care, and necropsy of the animal subject follow a model used in a previous study undertaken at this institution. ${ }^{38}$ The study was approved by the Institutional Animal Care and Use Committee (IACUC) at Baylor College of Dentistry.

\section{SURGICAL PROCEDURES}

In order to create a ridge defect, four premolars ${ }^{1-4}$ were extracted on each side of the mandible, under general anesthesia. Extractions were performed using a flap approach to allow sectioning of the teeth. The flaps were reapproximated and sutured with 4.0 polyglactin 910 (Vicryl $^{\mathrm{TM}}$, Ethicon, Johnson and Johnson, Cincinnati, OH, USA) suture material. Eight weeks following extractions, the second phase of the study was initiated to create the alveolar bone defects in the mandible. The first three defects in each animal were allocated to receive each of the three treatment groups (CM, CS, Ctrl) with the fourth defect randomly allocated to one of the three groups, ensuring a uniform distribution of groups among all animals. Following general anesthesia, a midcrestal incision was made across the edentulous area from mesial to distal, with intrasulcular extensions of the incision at teeth immediately adjacent to the site. Mucoperiosteal flaps were reflected on the buccal and lingual aspect of the site using an envelope flap. After exposing the alveolar ridge, a semicircular cut was made in the crest of the ridge using a $6.0 \mathrm{~mm}$ diameter trephine bur on a dental handpiece at a speed of approximately $600 \mathrm{rpm}$ irrigated with sterile saline. All defects were created to a standardized morphology, with proportions of $3.0 \mathrm{~mm}$ in the buccolingual dimension, $6.0 \mathrm{~mm}$ in the mesiodistal dimension, and $6.0 \mathrm{~mm}$ in the apico-occlusal dimension (Figs 1A to D). The center of the most distal site was located $6 \mathrm{~mm}$ mesial to the $1 \mathrm{st}$ molar and the center of the next site was located $9 \mathrm{~mm}$ mesial to the most distal site.

In the experimental sites, autogenous bone graft was placed into the defect prior to the placement of the barrier. The graft consisted of boneharvested from the mandible using an osseous collection device (SafeScraper ${ }^{\mathrm{TM}}$, BioMet 3i, 

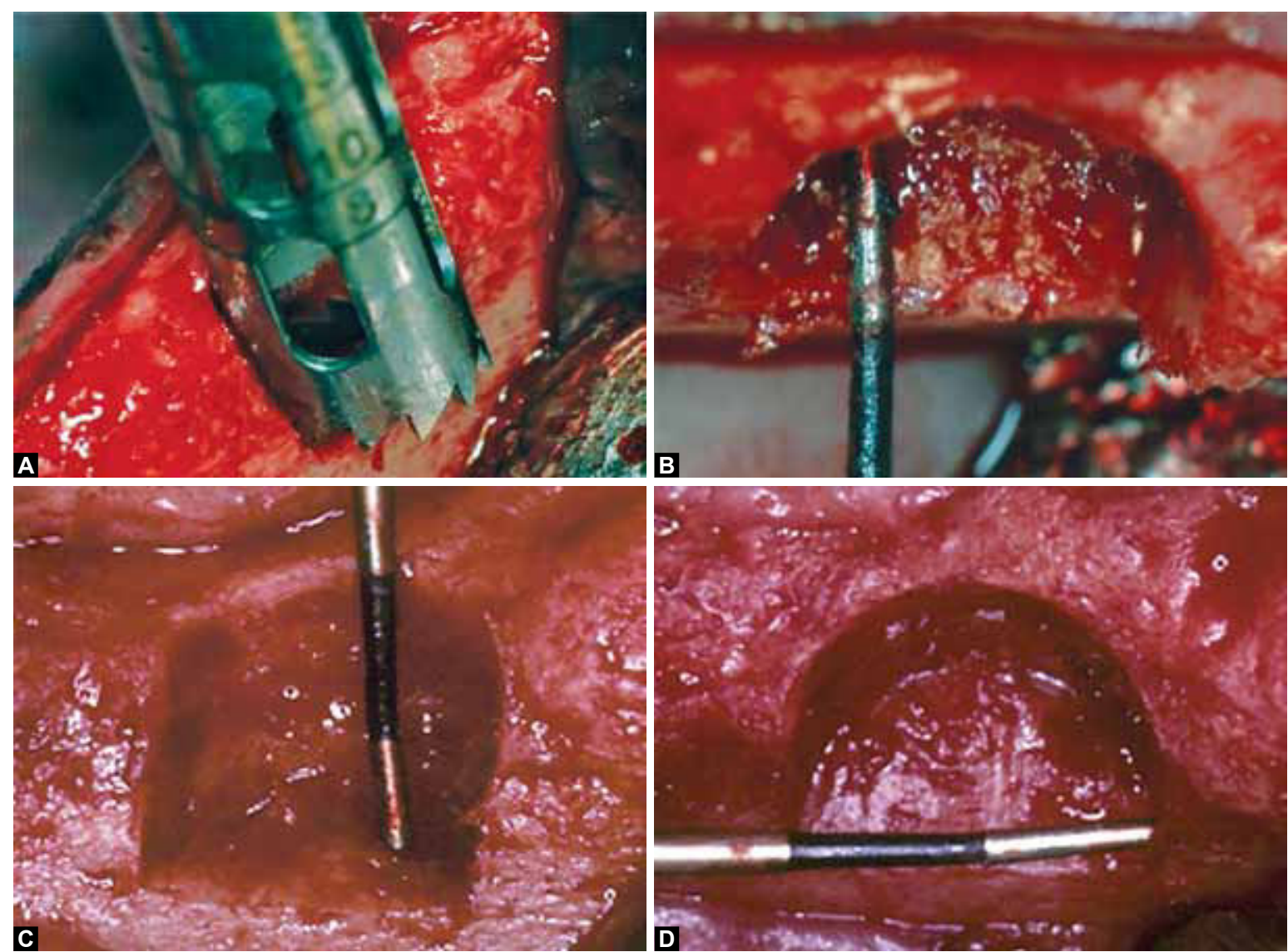

Figs 1A to D: Alveolar ridge defects were created in the mandible using a $6.0 \mathrm{~mm}$ trephine $(\mathrm{A})$, with a depth of $3 \mathrm{~mm}(\mathrm{~B})$, a height of $6 \mathrm{~mm} \mathrm{(C)}$ and a width of $6 \mathrm{~mm}(\mathrm{D})$

Palm Beach Gardens, FL, USA). The bone was collected from the exposed mandible at least $15 \mathrm{~mm}$ away from the experimental and control sites. The trephined bone obtained after defect creation also provided donor autogenous graft material. Prior to placement in the defect, the bone graft was mixed together with blood collected from the surgical site. The graft was then covered with either, calcium sulfate, CS (Calcigen ${ }^{\mathrm{TM}}$ Oral, BioMet 3i, Palm Beach Gardens, FL, USA) putty or a bovine collagen membrane, CM(Ossix ${ }^{\mathrm{TM}}$, BioMet 3i, Palm Beach Gardens, FL, USA) depending on site allocation (Figs $2 \mathrm{~A}$ and B). The $\mathrm{CM}$ was hydrated in sterile saline and placed over the grafted site with at least $3 \mathrm{~mm}$ overlap onto adjacent bone. The CS putty was prepared by mixing a setting solution consisting primarily of distilled water with CS dihydrate powder. The CS mixture was allowed to thicken to a putty-like consistency, then shaped and placed over the grafted site. Control sites were operated using the same surgical protocol that was used for experimental sites, including flap reflection and defect creation; however, no grafting or augmentation of the sites were performed.

Cortical perforations were created in the coronal aspect of the alveolar ridge using a \#2 round bur. Perforations were placed $1 \mathrm{~mm}$ lingual to the center of the surgically-created defect followed by deposition of India ink dye. This tattoo served as a reference point for iden- tification when sectioning the specimens for histological analysis. The mucoperiosteal flaps were reapproximated to obtain primary wound closure and sutured with 4.0 polyglactin 910 suture material, with periosteal releasing incisions made as needed to assure minimal flap tension.

Twelve weeks following defect creation, all animals were euthanized under general anesthesia and the mandibles were resected en bloc with an oscillating autopsy saw, immersed in $70 \%$ ethanol and submitted for histological processing and analysis. Bone regeneration was evaluated by measuring defect fill in terms of area, percent bone gain, width and cortical bone thickness.

\section{HISTOLOGICAL ANALYSIS}

Individual sites were sectioned and bisected with a rotary diamond blade with a thickness of $0.5 \mathrm{~mm}$ in a buccolingual orientation using the crestal India ink tattoos and 1st molar teeth as reference points. Each specimens was prepared for histological analysis, 10 sections of each site were obtained in a longitudinal axis from facial to lingual. The sections were stained with alizarin red, which stains osteoid and mineralized bone tissues red.

Image acquisition of the entire section, including the complete buccal defect area, was accomplished using a digital camera attached to a light microscope 

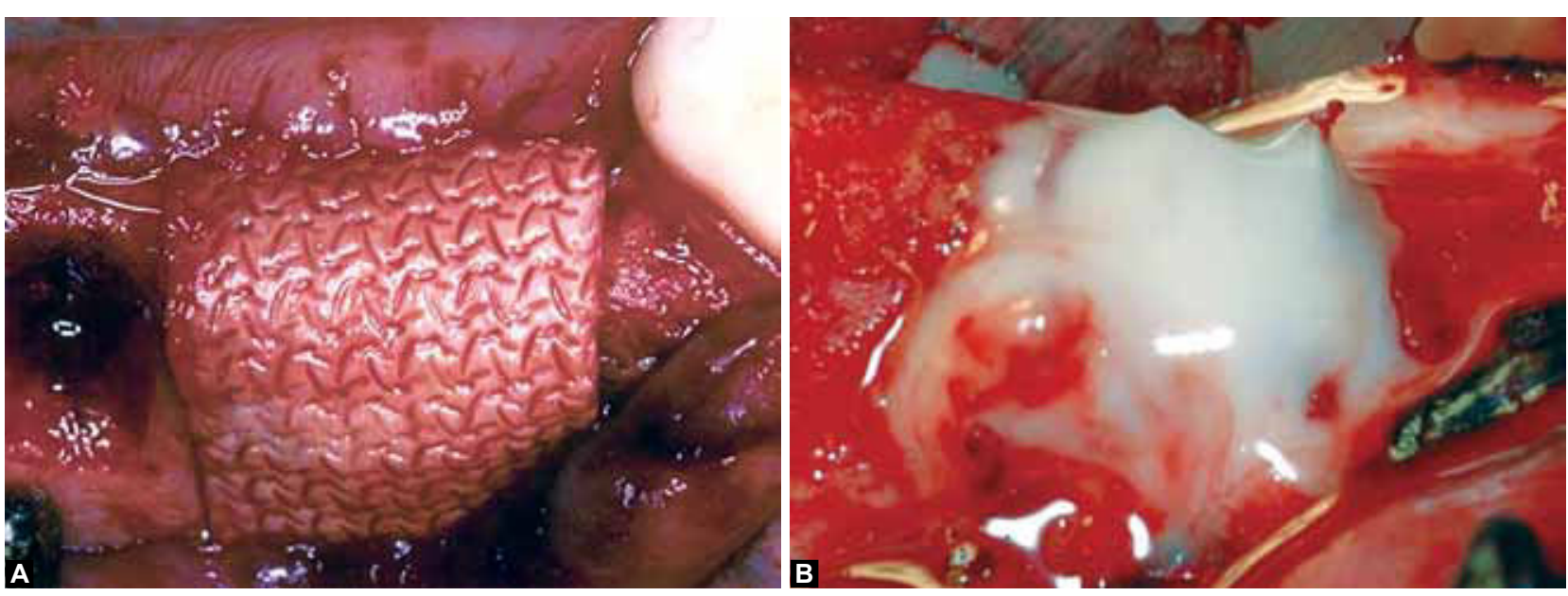

Figs $2 A$ and $B$ : Defects were grafted with autogenous bone and covered with either CM (A) or CS (B)

(Olympus DP-12, Olympus America, Center Valley, PA, USA). A calibrated scale was created using the image capture software that could be superimposed over the digital image. The scale had a dimension of $3 \mathrm{~mm}$ by $6 \mathrm{~mm}$, corresponding to the dimensions of the surgically created defect (Figs 3A and B). Positioning of the scale was accomplished using the alveolar crest and buccal cortex as reference points, with an orientation of $3 \mathrm{~mm}$ buccolingual and $6 \mathrm{~mm}$ coronoapical. This allowed for more precise location of the defect during histological analysis.

The defect regions were analyzed histomorphometrically using an image analysis software program (MetaMorph, Molecular Devices Corp, Downingtown, PA, USA), (Figs 4A to D). All histomorphometric analysis was done without knowledge of the treatment assignment of the sections. Using the applied $3 \times 6 \mathrm{~mm}$ scale as a reference, a calibration was performed ( 208 pixels $=1 \mathrm{~mm}$ ). The primary variable measured within the defect was the total regenerated area. This was accomplished by tracing the perimeter of the defect with the borders consisting of the buccal cortex and the applied scale (43264 pixels = $1 \mathrm{~mm}^{2}$ ). Additionally, the bone fill for the entire defect was measured by outlining each individual segment of bone within the defect, with the summation of each segment equaling the total area of bone regenerate. The sum total area of bone regenerate for each defect was divided by a fixed measurement, specifically the area of a $3 \times 6 \mathrm{~mm}$ rectangle, yielding a percentage of bone fill in each defect. Use of the $3 \times 6 \mathrm{~mm}$ rectangle as a standardized area was designated to equilibrate all sites for group comparison, as no one defect was morphologically identical to another.

Another parameter included measurement of the width of bone regenerate within the defect, which was obtained by measuring the linear distance from the buccal cortex of the defect inward to the border of the applied scale. This distance was calculated at the crest of the defect, coronal 1/3rd ( $2 \mathrm{~mm}$ apical of the crest), apical $1 / 3 \mathrm{rd}$ ( $4 \mathrm{~mm}$ apical of the crest), and at the base of the defect according to the applied reference scale. Analysis of the thickness of the regenerated buccal cortex within the defect was also accomplished. This measurement was obtained by identifying the area of greatest and least thickness within the cortex, then calculating the linear distance of thickness at each site to determine the range of cortical bone thickness.

\section{STATISTICAL ANALYSIS}

The data was entered into a statistical software program (SPSS, Chicago, IL, USA). Descriptive statistics were calculated for each site, including total area of regenerate, the percentage of bone regenerate as compared to the standard $3 \times 6 \mathrm{~mm}$ reference area, the width of regenerate at the crest, coronal third, apical third, and base of the defects, and the maximum and minimum width of the regenerated buccal cortex. All four measurements were compared with an analysis of variance (ANOVA) between study groups with the defect site as the individual unit of observation. ANOVA was also performed for each of the four variables to determine if the significance of possible confounding variables including whether defect sites were influenced by animal. A student's t-test was performed to determine whether anterior-posterior defect location had any influence on regeneration within the defects.

\section{RESULTS}

All animals successfully completed the initial phases of treatment consisting of bilateral mandibular premolar extractions, followed by ridge augmentation procedures after 8 weeks of healing. One animal died 7 days following alveolar ridge augmentation procedures, suffering from acute renal failure secondary to undiagnosed pancreatic cancer (necropsy report). Sites allocated within this animal included two sites treated with a $\mathrm{CM}$, one treated with CS, and one site designated as a control. 
Individual sites from this animal were submitted for histological processing and analysis of the specimens was performed; however, data from these sites were only evaluated and not included in the statistical analysis (Figs 3A and B). The other five animals completed the study without complications.

Examination of the surgical sites at 1 week postsurgically revealed no dehiscence of soft tissue or membrane exposure, with maintenance of primary wound healing over all defect sites in all animals. Following necropsy and tissue fixation, histological analysis of individual sites revealed regeneration of soft and hard tissues within all surgically created defects after 12 weeks of healing
(Figs 5A to C). All sections were appropriately stained and mounted for histological analysis and comparison.

The descriptive statistics revealed a similar mean and median for all outcomes, indicating the presence of a normal distribution. Thus, the sample met the requirements for parametric analysis.

A comparison between groups revealed a greater mean area of regenerated bone in sites treated using $\mathrm{CM}$ and bone graft $\left(12.2 \mathrm{~mm}^{2}\right)$ compared to sites treated using CS and bone graft $\left(11.6 \mathrm{~mm}^{2}\right)$ or control sites $\left(11.9 \mathrm{~mm}^{2}\right)$. However, an analysis of variance found no statistical significance $(\mathrm{p}=0.875)$ in these differences (Table 1).
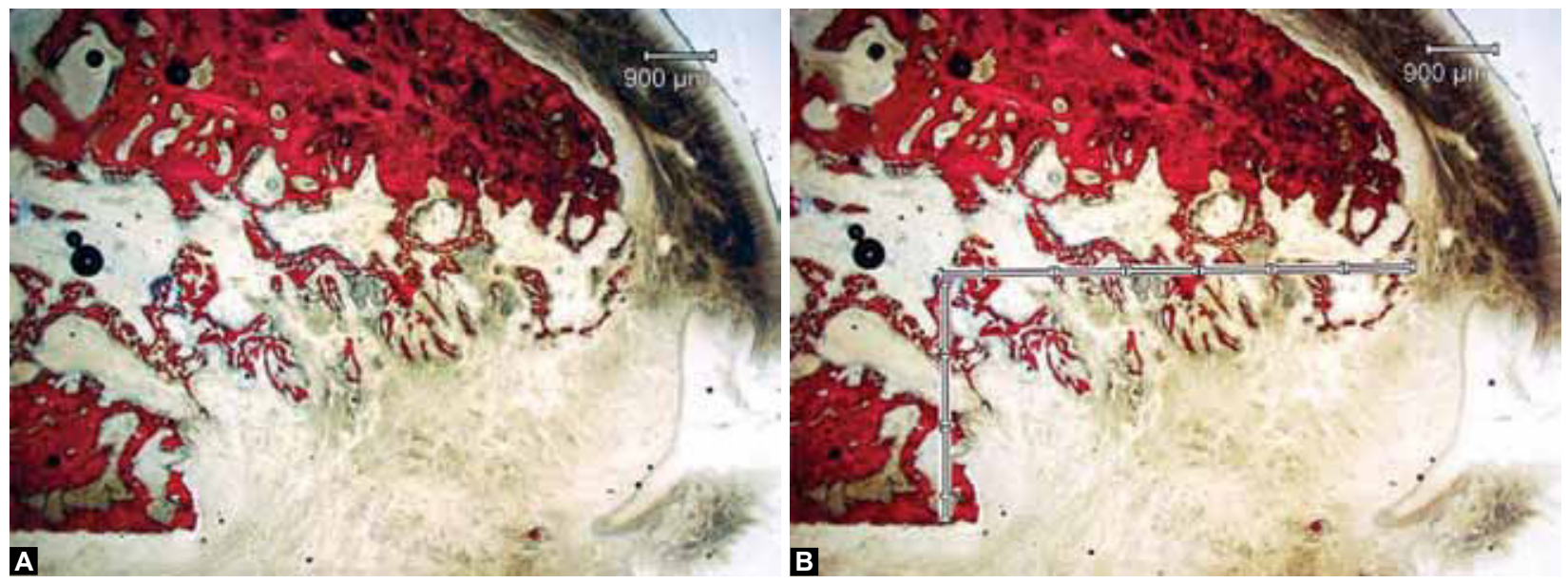

Figs $3 A$ and B: Histology of the site 1 week after defect creation without $(A)$ and with $(B)$ reference scale (alizarin red stain; original magnification $\times 0.625$ )
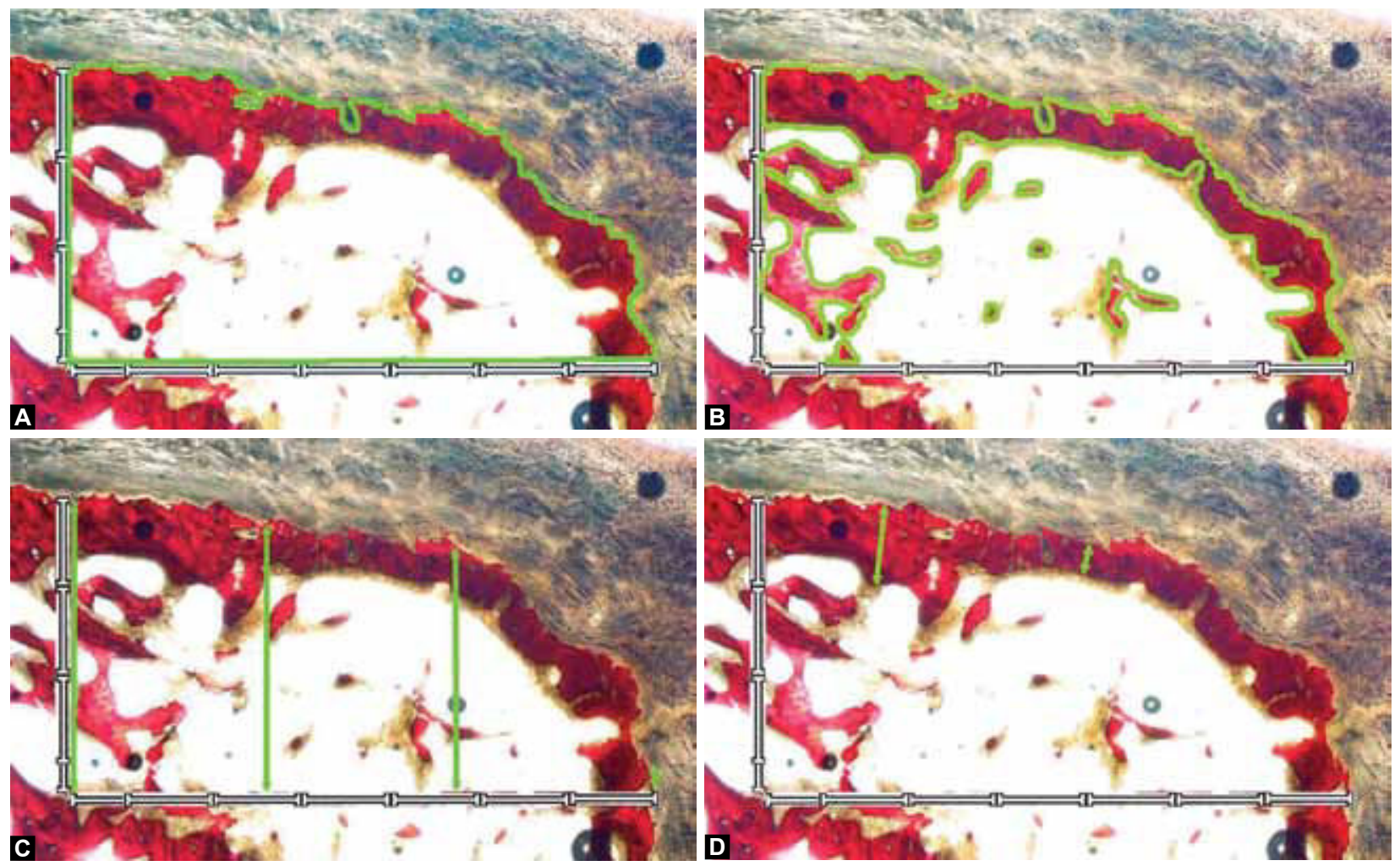

Figs 4A to D: Histomorphometric analysis of specimens for total area (A), percent bone (B), width at crest, coronal 1/3rd, apical $1 / 3 \mathrm{rd}$, and base of the defect (C) and range of cortical bone thickness (D). Green line indicates where measurements were taken 
The percentage of bone within the defects as related to a standardized area was found to be greatest in sites treated with CS and bone graft (38.2\%), followed closely by control sites $(37.9 \%)$ and sites treated with $\mathrm{CM}$ and bone graft $(36.3 \%)$, although no statistically significant difference was detected between groups using ANOVA $(\mathrm{p}=0.942)$.

Measurement of cortical bone thickness revealed a range of 0.49 to $1.43 \mathrm{~mm}$ for control sites and 0.56 to $1.38 \mathrm{~mm}$ for CS sites. Defects treated with CM and bone graft demonstrated the thinnest buccal cortex, with a range of 0.41 to $1.06 \mathrm{~mm}$. Although these measurements demonstrated the most variability of all evaluated parameters, no statistically significant differences were found between groups using ANOVA for maximum $(p=0.347)$ and minimum cortical bone thickness $(\mathrm{p}=0.688)$.

An evaluation of the width of regenerated bone at four different levels revealed little differences between groups at all points of measurement (Table 2). All sites regardless of treatment demonstrated little horizontal bone fill at the crest, with a mean distance of $0.33 \mathrm{~mm}$ at the crest for all three groups. Width of bone growth at the crestal level was not significantly different between groups according to ANOVA $(p=1.00)$. Horizontal fill at the coronal $1 / 3$ rd of the site was greatest in defects treated with $\mathrm{CM}$ and bone graft $(1.62 \mathrm{~mm})$ and control defects $(1.61 \mathrm{~mm})$, and slightly less in those treated with CS and bone graft $(1.48 \mathrm{~mm})$; however, no statistically significant differences were found between groups using ANOVA $(p=0.879)$. In the apical $1 / 3 \mathrm{rd}$ of the site, the greatest horizontal fill was again seen in the CM group $(2.30 \mathrm{~mm})$ followed by control sites $(2.22 \mathrm{~mm})$ and CS sites $(2.08 \mathrm{~mm})$, although no statistically significant differences were seen using ANOVA ( $p=0.626)$. Finally, all treatment groups

Table 1: Regenerated bone area, percent bone fill and cortical bone thickness

\begin{tabular}{llll}
\hline Group & $\begin{array}{l}\text { Area } \\
\left(\mathrm{mm}^{2}\right)\end{array}$ & $\begin{array}{l}\text { Percent } \\
\text { bone }(\%)\end{array}$ & $\begin{array}{l}\text { Cortical bone } \\
\text { thickness }(\mathrm{mm})\end{array}$ \\
\hline Collagen membrane & 12.1 & 36.3 & $0.41-1.06$ \\
Calcium sulfate & 11.6 & 38.2 & $0.56-1.38$ \\
Control & 11.9 & 37.9 & $0.49-1.43$ \\
\hline
\end{tabular}

Table 2: Width of regenerated bone within the defect

\begin{tabular}{lllll}
\hline Group & $\begin{array}{l}\text { Crest } \\
(\mathrm{mm})\end{array}$ & $\begin{array}{l}\text { Coronal } \\
1 / 3 r d(\mathrm{~mm})\end{array}$ & $\begin{array}{l}\text { Apical 1/3rd } \\
(\mathrm{mm})\end{array}$ & $\begin{array}{l}\text { Base } \\
(\mathrm{mm})\end{array}$ \\
\hline $\begin{array}{l}\text { Collagen } \\
\text { membrane }\end{array}$ & 0.33 & 1.62 & 2.30 & 2.85 \\
$\begin{array}{l}\text { Calcium } \\
\text { sulfate }\end{array}$ & 0.33 & 1.48 & 2.08 & 2.85 \\
Control & 0.33 & 1.61 & 2.22 & 2.82 \\
\hline
\end{tabular}
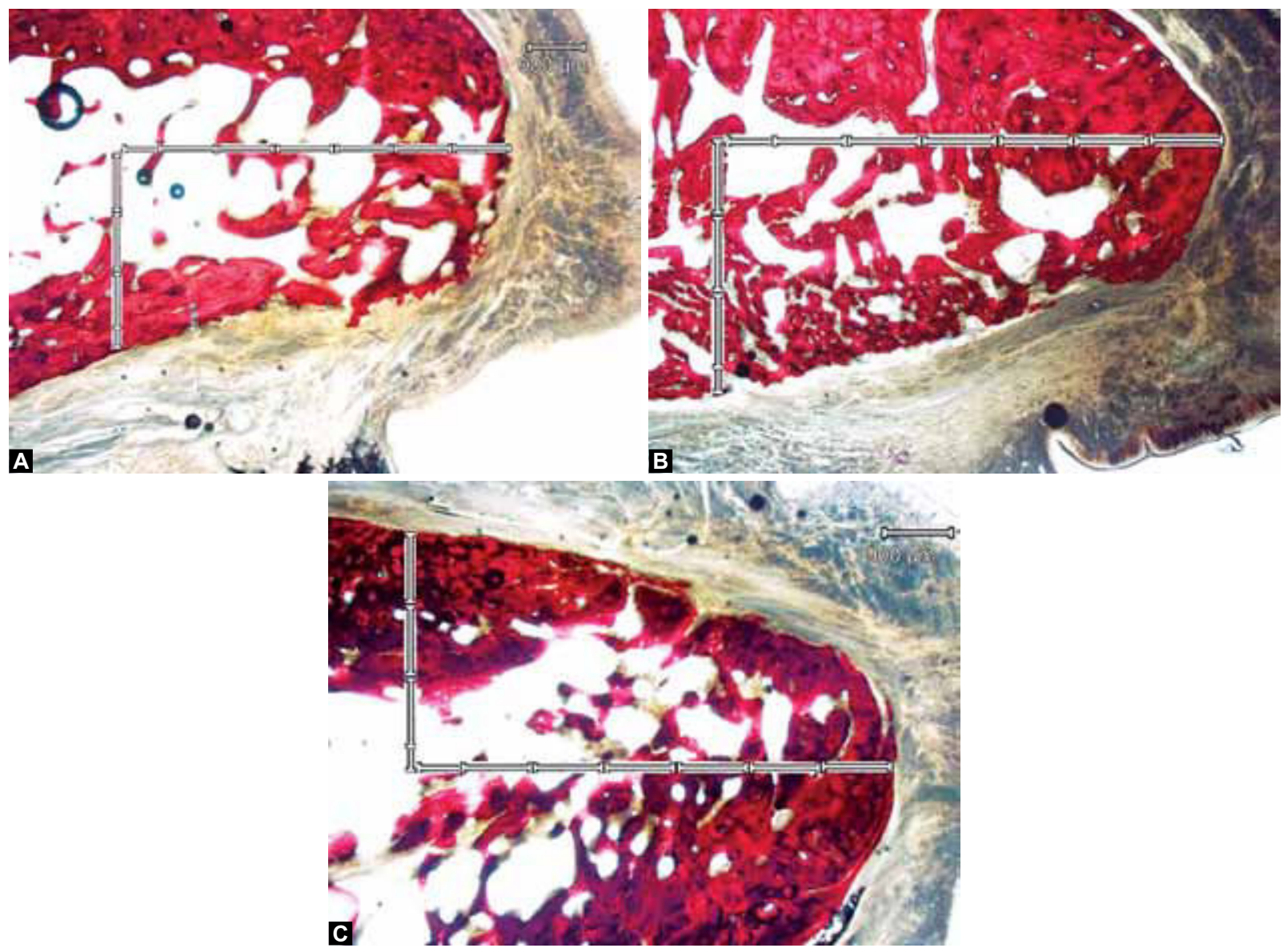

Figs 5A to C: Histomorphometry of three sites 12 weeks after defect creation. These sites were treated using CM (A) or CS (B) as well as a nongrafted control specimen (C) 
demonstrated similar horizontal fill at the base of the defects, with a fill of $2.85 \mathrm{~mm}$ for the CM and CS groups and $2.82 \mathrm{~mm}$ for control sites. No statistically significant differences were found between any of the groups using ANOVA ( $p=0.867)$.

An analysis of variance evaluating differences between animals demonstrated no statistically significant differences for most parameters, including total area, horizontal width at all levels (crest, cortical 1/3rd, apical 1/3rd, base), and percent bone. Cortical bone thickness in terms of mean maximum thickness was found to be significantly greater for one animal (1.97 mm, $\mathrm{p}=0.001)$; however, mean minimum thickness was not significantly different $(p=0.089)$.

Differences between sites in terms of location were analyzed using a student's t-test. Results demonstrated no statistically significant differences between all parameters except percent bone, which was found to be $42.6 \%$ \pm 7.6 for mesial sites and $32.4 \% \pm 9.6$ for distal sites $(\mathrm{p}=$ $0.017)$.

\section{DISCUSSION}

This study evaluated bone regeneration in surgically created alveolar ridge defects in the canine mandible using autogenous bone grafts and barriers consistent with the principles of GBR. The canine model was chosen because of the potential for intersubject comparison, as well as the opportunity to control the defect size. Multiple measures of bone regeneration were evaluated from histologic sections, including the total area of bone regenerated within the surgically created defect. Measurements were made on four sites, specifically, the crest, coronal third, apical third and at the base of the defect. Our results showed similar amount of width regeneration for all groups (Table 2).

Regeneration within the defects was also measured in terms of percent bone gain. This measurement was taken by calculating the total area of bone particles within the defect and dividing that number by a standardized $3 \times 6 \mathrm{~mm}$ area; therefore, the percentage value does not reflect the actual percent bone regenerated within the defect itself. Comparison of percent bone to a standardized area was performed to eliminate variability seen within groups based on morphology of the regenerated defects. Percent bone regeneration was found to approximate $38 \%$ in sites treated with CS, which was only slightly greater than control sites (37.9\%) and sites treated using a collagen membrane $(36.3 \%)$.

Cortical bone thickness is an important clinical finding in regenerated bone, as the cortical bone provides primary stability for dental implants immediately after placement. Few studies quantified the thickness of cortical bone, but Lekholm and Zarb's classification of bone types emphasizes the thickness of cortical bone in relation to implant success in type IV bone, which is characterized by thin cortical bone and minimal trabecular bone. ${ }^{39}$ Albrektsson et al found that survival rates of machinedsurfaced titanium implants placed in type IV bone were significantly less than implants placed in types I, II or III bone. Therefore, it is particularly important for the clinician placing a dental implant in a previously grafted site to appreciate the thickness of the regenerated cortex as it may affect the course of treatment. For example, the clinician may choose to place the implant using a two-stage submerged approach instead of a one-stage transgingival approach if primary stability of the implant cannot be obtained.

In this study, the cortical bone thickness was greatest in nongrafted sites (Ctrl) with a range of 0.49 to $1.43 \mathrm{~mm}$. This was comparable to sites treated with CS, which exhibited a range of 0.56 to $1.38 \mathrm{~mm}$. Sites treated using a collagen membrane demonstrated the thinnest cortical bone of all treatment groups, with a range of 0.41 to $1.06 \mathrm{~mm}$ respectively. While the differences between the groups were small and not significantly different, there may be clinical implications associated with these measurements.

A reasonable explanation as to why cortical thickness was less in sites treated with the collagen membrane may lie in the occlusive nature of the barrier. The collagen membrane used in this study has shown to provide barrier function for up to 6 months in humans, and is visible in many of the histologic specimens taken from this study as well as others. ${ }^{19,20,24}$ The increased barrier function of this membrane was likely due to cross-linkage of the collagen fibers. ${ }^{40}$ Other studies reported poor regeneration using highly cross-linked collagen membranes, resulting in increased rates of flap dehiscence compared to low cross-linked membranes. ${ }^{41}$ Additionally, the occlusive nature of the membrane may reduce blood supply to the underlying graft as it prevents connective tissue from coming in contact with the grafted bone, which may limit the regeneration of the buccal cortex. This effect was not seen in control sites or sites treated with CS. Perhaps an increased healing period allowing for further degeneration of the membrane would have produced different results, such as an increased thickening of the buccal cortex in sites treated with a collagen membrane.

A possible explanation of why cortical thickness was greater in the control group could be due to an increased blood supply to control sites, as no barrier devices were placed. Increased cortical thickness in CS sites might be explained by a more rapid degradation of the barrier device, reported to be approximately 8 weeks, which would allow for an increased blood supply to the regenerating 
cortex as the CS resorbs. ${ }^{30}$ This study supports the findings of Sottosanti based on the fact that CS remnants were not detected in any of the histologic specimens.

Additional comparisons between individual sites were generally found to be not significantly different, with the exception of site location. In terms of percent bone, mesial sites were found to have an increased amount of regeneration over distal sites, on the order of 42.6 to $32.4 \%$. This difference was found to be statistically significant using a student's t-test. A possible explanation for this difference might be related to Lekholm and Zarb's classification of bone types. ${ }^{39}$ In their explanation of bone structure based on anatomical location, they found a decrease in trabecular bone density in a mesial to distal direction in the maxilla and mandible. While the distance between mesial and distal sites in this experiment was only $9 \mathrm{~mm}$, this could be sufficient to explain the difference in the bone percentage. Other considerations include the close proximity of the mesial defect to the mental foramina, of which this canine species has two bilaterally, which would provide a greater periosteal blood supply to a regenerating graft.

Other findings of this study include the lack of intraoral complications following surgical procedures. No soft tissue dehiscences were detected following augmentation in either the collagen membrane or CS groups, which confirm previous findings stating the biocompatibility of these materials. ${ }^{40,42}$ Additionally, all groups exhibited some regeneration, although regeneration within control sites was not found to be significantly different from the experimental sites. This was an unexpected finding, as most controlled studies of ridge augmentation have demonstrated a reduced regeneration within control sites compared with grafted and/or membrane treated sites. $^{43,44}$

An explanation for the profound regeneration within all sites may be related to the size of the surgically created defect. Design of the semicircular defect was chosen to simulate an atrophic ridge similar to what often is encountered when the buccal cortex is lost following tooth extraction. This study involved creation of a buccal ridge defect measuring $3 \times 6 \times 6 \mathrm{~mm}$ (buccolingual, coronoapical, mesiodistal). Other studies with surgically created defects have utilized larger defects. ${ }^{43-47}$ In a study of ridge augmentation in the canine, Seibert and Nyman created buccal ridge defects measuring $3.5 \times 7 \times$ $13 \mathrm{~mm}$ to compare GBR using membranes. The authors reported new bone formation in sites covered with a membrane after 90 days, with no new bone formation in sham-operated control sites. Von Arx et al created buccal ridge defects in the canine mandible with dimensions of $8 \times 10 \times 14 \mathrm{~mm}$ for the purpose of comparing GBR using various bone grafting materials with or without e-PTFE membranes. All sites exhibited regeneration within the defects after 6 months; however, no empty control defects were utilized. Within this study, no statistically significant differences were seen for bone regeneration achieved within experimental or control defects in terms of area, percent bone, width and cortical thickness, leading to the assumption that the dimensions of the defect used in this study were not large enough to allow for comparison of grafted vs nongrafted ridge defects (critical-sized defects). It is possible that creation of a larger size defect comparable to that utilized in the study by Seibert and Nyman may result in more pronounced differences between experimental and control groups. ${ }^{43}$ Perhaps the critical size for an alveolar ridge defect in the canine may be somewhere in between the size of the defect used in this study and those used in other studies.

The results from this study demonstrate that calcium sulfate is a biocompatible material that may be useful as an adjunctive grafting component in GBR procedures. It is unclear whether the regeneration achieved using CS is directly related to its barrier function or whether the CS merely stabilized the graft material during initial wound healing. Calcium sulfate has been used succes sfully as a binding agent for bone grafting material in ridge preservation and periodontal regenerative procedures, therefore, future research may address the capabilities of calcium sulfate as a bone graft binder and as a barrier in combination for ridge augmentation procedures.

\section{ACKNOWLEDGMENTS}

The authors would like to thank Mrs Jo Taylor for histologic processing and Mr Gerald Hill and Ms Priscilla Gillespie for assistance in the Animal Resource Unit. This study was supported by a grant and materials donated by BioMet 3i.

\section{REFERENCES}

1. Lekovic V, Camargo PM, Klokkevold PR, et al. Preservation of alveolar bone in extraction sockets using bioabsorbable membranes. J Periodontol 1998;69(9):1044-1049.

2. Fugazzotto PA. Treatment options following single-rooted tooth removal: a literature review and proposed hierarchy of treatment selection. J Periodontol 2005;76(5):821-831.

3. Iasella JM, Greenwell H, Miller RL, et al. Ridge preservation with freeze-dried bone allograft and a collagen membrane compared to extraction alone for implant site development: a clinical and histologic study in humans. J Periodontol 2003; 74(7):990-999.

4. Nemcovsky CE, Serfaty V. Alveolar ridge preservation following extraction of maxillary anterior teeth. Report on 23 consecutive cases. J Periodontol 1996;67(4):390-395. 
5. Buser D, Bragger U, Lang NP, Nyman S. Regeneration and enlargement of jaw bone using guided tissue regeneration. Clin Oral Implants Res 1990;1(1):22-32.

6. Becker W, Becker BE, McGuire MK. Localized ridge augmentation using absorbable pins and e-PTFE barrier membranes: a new surgical technique. Case reports. Int J Periodontics Restorative Dent 1994;14(1):48-61.

7. Von Arx T, Buser D. Horizontal ridge augmentation using autogenous block grafts and the guided bone regeneration technique with collagen membranes: a clinical study with 42 patients. Clin Oral Implants Res 2006;17(4):359-366.

8. Fiorellini JP, Nevins ML. Localized ridge augmentation/ preservation: a systematic review. Ann Periodontol 2003;8(1): 321-327.

9. McAllister BS. Histologic and radiographic evidence of vertical ridge augmentation utilizing distraction osteogenesis: 10 consecutively placed distractors. J Periodontol 2001;72(12):17671779.

10. Marchetti C, Corinaldesi G, Pieri F, Degidi M, Piattelli A. Alveolar distraction osteogenesis for bone augmentation of severely atrophic ridges in 10 consecutive cases: a histologic and histomorphometric study. J Periodontol 2007;78(2):360366.

11. Howell TH, Fiorellini J, Jones A, et al. A feasibility study evaluating rhBMP-2/absorbable collagen sponge device for local alveolar ridge preservation or augmentation. Int J Periodontics Restorative Dent 1997;17(2):124-139.

12. Cochran DL, Jones AA, Lilly LC, Fiorellini JP, Howell H. Evaluation of recombinant human bone morphogenetic protein-2 in oral applications including the use of endosseous implants: 3-year results of a pilot study in humans. J Periodontol 2000;71(8):1241-1257.

13. Bunyaratavej $\mathrm{P}$, Wang HL. Collagen membranes: a review. J Periodontol 2001;72(2):215-229.

14. Wang HL, Greenwell H, Fiorellini J, et al. Periodontal regeneration. J Periodontol 2005;76(9):1601-1622.

15. Wang HL, MacNeil RL. Guided tissue regeneration. Absorbable barriers. Dent Clin North Am 1998;42(3):505-522.

16. Murphy KG. Postoperative healing complications associated with gore-tex periodontal material. Part II. Effect of complications on regeneration. Int J Periodontics Restorative Dent 1995;15(6):548-561.

17. Murphy KG. Postoperative healing complications associated with gore-tex periodontal material. Part I. Incidence and characterization. Int J Periodon Restorative Dent 1995;15(4): 363-375.

18. Machtei EE. The effect of membrane exposure on the outcome of regenerative procedures in humans: a meta-analysis. J Periodontol 2001;72(4):512-516.

19. Friedmann A, Strietzel FP, Maretzki B, Bernimoulin JP. Histological assessment of augmented jaw bone utilizing a new collagen barrier membrane compared to a standard barrier membrane to protect a granular bone substitute material. Clin Oral Implants Res 2002;13(6):587-594.

20. Friedmann A, Strietzel FP, Maretzki B, Pitaru S, Bernimoulin JP. Observations on a new collagen barrier membrane in 16 consecutively treated patients. Clinical and histological findings. J Periodontol 2001;72(11):1616-1623.

21. Minabe M, Kodama T, Kogou T, et al. Different crosslinked types of collagen implanted in rat palatal gingiva. J Periodontol 1989;6(1):35-43.
22. Cortellini P, Pini Prato G, Tonetti MS. Periodontal regeneration of human intrabony defects with bioresorbable membranes: a controlled clinical trial. J Periodontol 1996;67(3):217-223.

23. Blumenthal NM. A clinical comparison of collagen membranes with e-PTFE membranes in the treatment of human mandibular buccal class II furcation defects. J Periodontol 1993;64(10):925-933.

24. Ersanli S, Olgac V, Leblebicioglu B. Histologic analysis of alveolar bone following guided bone regeneration. J Periodontol 2004;75(5):750-756.

25. Sottosanti JS. Calcium sulfate: a biodegradable and biocompatible barrier for guided tissue regeneration. Compend Contin Educ Dent 1992;8:226-234.

26. Orsini M, Orsini G, Benlloch D, et al. Comparison of calcium sulfate and autogenous bone graft to bioabsorbable membranes plus autogenous bone graft in the treatment of intrabony periodontal defects: a split-mouth study. J Periodontol 2001;72(3):296-302.

27. Peltier LF. The use of plaster of Paris to fill large defects in bone: a preliminary report 1959. Clin Orthop 2001;382:3-5.

28. Urban RM, Turner TM, Hall DJ, Infranger S, Cheema N, Lim $\mathrm{TH}$. Healing of large defects treated with calcium sulfate pellets containing demineralized bone matrix particles. Orthopedics 2003;26(5 suppl):s581-s585.

29. Payne JM, Cobb CM, Rapley JW, Killoy WJ, Spencer P. Migration of human gingival fibroblasts over guided tissue regeneration barrier materials. J Periodontol 1996;67(3):236-244.

30. Sottosanti JS. Aesthetic extractions with calcium sulfate and the principles of guided tissue regeneration. Pract Periodontics Aesthet Dent 1993;5(5):61-69.

31. Strocchi R, Orsini G, Iezzi G, et al. Bone regeneration with calcium sulfate: evidence for increased angiogenesis in rabbits. J Oral Implant 2002;28:273-278.

32. Vance GS, Greenwell H, Miller RL, Hill M, Johnston H, Scheetz JP. Comparison of an allograft in an experimental putty carrier and a bovine-derived xenograft used in ridge preservation: a clinical and histologic study in humans. Int J Oral Maxillofac Implants 2004;19(4):491-497.

33. Guarnieri R, Pecora G, Fini M, et al. Medical grade calcium sulfate hemihydrate in healing of human extraction sockets: clinical and histological observations at 3 months. J Periodontol 2004;75(6):902-908.

34. De Leonardis D, Pecora GE. Augmentation of the maxillary sinus with calcium sulfate: one-year clinical report from a prospective longitudinal study. Int J Oral Maxillofac Implants 1999;14(3):869-878.

35. Yoshikawa G, Murashima Y, Wadachi R, Sawada N, Suda $\mathrm{H}$. Guided bone regeneration (GBR) using membranes and calcium sulphate after apicectomy: a comparative histomorphometrical study. Int Endod J 2002;35(9):255-263.

36. Al Ruhaimi KA. Bone graft substitutes: a comparative qualitative histologic review of current osteoconductive grafting materials. Int J Oral Maxillofac Implants 2001;16(1):105-114.

37. Pecora G, De Leonardis D, Ibrahim N, Bovi M, Cornelini R. The use of calcium sulphate in the surgical treatment of a through and through periradicular lesion. Int Endod J 2001; 34(3):189-197.

38. Hodges NE, Perry M, Mohamed W, Hallmon WW, Rees T, Opperman LA. Distraction osteogenesis versus autogenous onlay grafting. Part II: biology of regenerate and onlay bone. Int J Oral Maxillofac Implants 2006;21(2):237-244.

39. Albrektsson T, Zarb G, Worthington P, Eriksson AR. The longterm efficacy of currently used dental implants: a review and 
proposed criteria of success. Int J Oral Maxillofac Implants 1986;1(1):11-25.

40. Rothamel D, Schwarz F, Sager M, Herten M, Sculean A, Becker J. Biodegradation of differently cross-linked collagen membranes: an experimental study in the rat. Clin Oral Implants Res 2005;16(3):369-378.

41. Crigger M, Bogle GC, Garrett S, Gantes BG. Repair following treatment of circumferential periodontal defects in dogs with collagen and expanded polytetrafluoroethylene barrier membranes. J Periodontol 1996;67(4):403-413.

42. Sidqui M, Collin P, Vitte C, Forest N. Osteoblast adherenceand resorption activity of isolated osteoclasts on calxium sulphate hemihydrate. Biomaterials 1995;16(17):1327-1332.

43. Seibert J, Nyman S. Localized ridge augmentation in dogs: a pilot study using membranes and hydroxyapatite. J Periodontol 1990;61(3):157-165.
44. Smukler H, Barboza EP, Burliss C. A new approach to regeneration of surgically reduced alveolar ridges in dogs: a clinical and histologic study. Int J Oral Maxillofac Implants 1995;10(5):537-551.

45. von Arx T, Cochran DL, Schenk RK, Buser D. Evaluation of a prototype trilayer membrane (PTLM) for lateral ridge augmentation: an experimental study in the canine mandible. Int J Oral Maxillofac Surg 2002;31(2):190-199.

46. Fritz ME, Jeffcoat MK, Reddy M, et al. Guided bone regeneration of large mandibular defects in a primate model. J Periodontol 2000;71(9):1484-1491.

47. von Arx T, Cochran DL, Hermann JS, Schenk RK, Buser D. Lateral ridge augmentation using different bone fillers and barrier membrane application. A histologic and histomorphometric pilot study in the canine mandible. Clin Oral Implants Res 2001;12(3):260-269. 